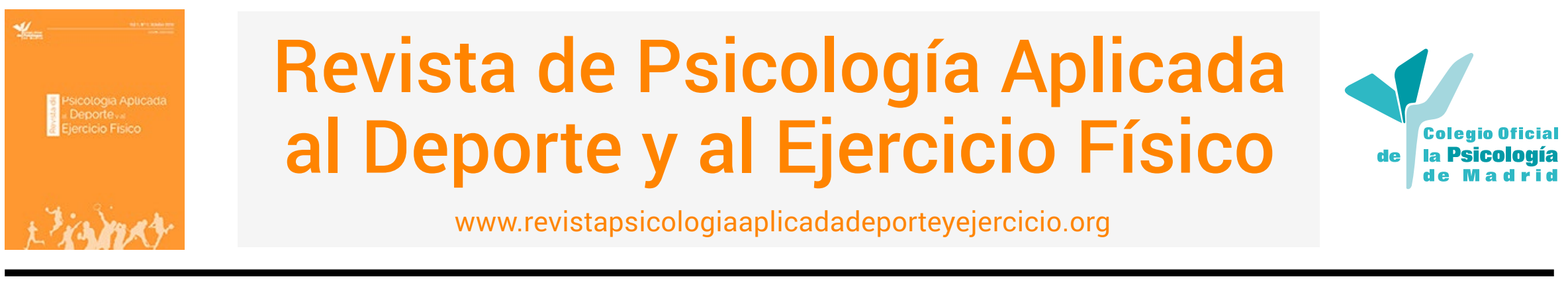

\title{
Esfuerzo físico y procesos atencionales en el deporte
}

\author{
Pablo Jodra ${ }^{1}$, María Ángeles Galera ${ }^{2}$, Omar Estrada ${ }^{3}$ y Raúl Domínguez ${ }^{4}$ \\ 'Universidad de Alcalá, España. ${ }^{2}$ Universidad Autónoma de Madrid, España. ${ }^{3} \mathrm{CEU}$ Cardenal Spínola de Sevilla, España. ${ }^{4}$ Universidad \\ Isabel I de Burgos, España
}

RESUMEN: Los procesos cognitivos básicos son aspectos fundamentales en el ámbito deportivo, pero a la hora de su correcto funcionamiento es preciso considerar el estado fatiga derivado de un esfuerzo físico. Los factores que contribuyen a la fatiga se derivan tanto del esfuerzo físico, como también de la carga mental concomitante y de los resultados de la tarea que se está realizando. En la actualidad no existe consenso sobre cómo afecta la realización de un esfuerzo físico en el rendimiento cognitivo. El objetivo de este trabajo fue comprobar si la realización de un esfuerzo físico de alta intensidad tiene influencia sobre el rendimiento cognitivo en deportistas entrenados. Se analizaron las diferencias entre los valores encontrados con la aplicación de la prueba de Toulouse-Pierón en línea base y tras una prueba de esfuerzo físico como es test de Wingate, en una muestra de 9 deportistas de élite de modalidad de combate. Los resultados indican diferencias significativas en las puntuaciones entre las dos fases de estudio $(t=-8.014 ; p<.001)$, mostrando mejores resultados del nivel atencional después de una prueba de esfuerzo. Se concluye que, los deportistas entrenados, cuando su organismo está sometido a esfuerzos de máxima intensidad, aumentan el rendimiento cognitivo en pruebas que requieren atención y rapidez mental.

PALABRAS CLAVES: Atención, Rendimiento cognitivo, Esfuerzo físico.

\section{Physical effort and attention processes in sport}

ABSTRACT: The basic cognitive processes are essential aspects in the sports field, but their adequate functioning depends on the state of fatigue derived from a physical effort. Factors contributing to fatigue derive from both physical exertion and concomitant mental load, and also from the results of the task being performed. There is currently no consensus as to how performance of a physical affects cognitive performance. The goal of this study was to verify whether a high intensity physical effort has an influence on the cognitive performance in trained athletes. Differences between the values found with the Toulouse-Pieron test at baseline and after a physical stress test such as the Wingate test were analysed in a sample of 9 elite athletes practising combat sports. The results suggest significant differences in the scores between the two study phases $(t=-8.014 ; p<.001)$, with better results in attention level after a stress test. It is concluded that trained athletes, when their body is subjected to maximum intensity efforts, increase their cognitive performance in tests that require attention and mental speed.

KEYWORDS: Attention, cognitive performance, physical effort.

\section{Esforço físico e processos de atenção no desporto}

RESUMO: Os processos cognitivos básicos são aspetos fundamentais no âmbito desportivo, mas em termos do seu correto funcionamento é necessário considerar o estado de fadiga decorrente de um esforço físico. Os fatores que contribuem para a fadiga resultam tanto do esforço físico, como da carga mental concomitante e dos resultados da tarefa que está a ser realizada. Atualmente, não há consenso sobre como isto afeta a realização de um esforço físico no desempenho cognitivo. O objetivo deste trabalho foi verificar

Pablo Jodra es Psicólogo en la Universidad de Alcalá.

María Ángeles Galera es Psicóloga en la Universidad Autónoma de Madrid.

Omar Estrada es Psicólogo en el CEU Cardenal Spínola de Sevilla.

La correspondencia sobre este artículo debe enviarse a Pablo Jodra al Departamento de Ciencias de la Educación, Universidad de Alcalá. Alcalá de Henares (España) 
se a realização de um esforço físico de alta intensidade influencia o desempenho cognitivo em atletas que treinam. Foram analisadas as diferenças entre os valores apresentados com base na aplicação do teste de Toulouse-Pieron na avaliação inicial e após uma prova de esforço físico, como o teste de Wingate, numa amostra de 9 atletas de elite de modalidades de combate. Os resultados indicavam diferenças significativas nas pontuações entre as duas fases do estudo ( $t=-8$ 014; $p<, 001)$, apresentando melhores resultados do nível de atenção após uma prova de esforço. Conclui-se que os atletas que treinam, registam um aumento no desempenho cognitivo em testes que requerem atenção e velocidade mental, quando os seus corpos são submetidos a esforços de intensidade máxima.

PALAVRAS-CHAVE: atenção, desempenho cognitivo, esforço físico.

Artículo recibido: 02/09/2019 | Artículo aceptado: 24/10/2019

Los procesos cognitivos básicos son los encargados de procesar la información del ambiente, mantenerla y dar una respuesta acorde (Santiago, Tornay, Gómez y Elosúa, 2006). En el mecanismo de atención, se filtra la información concreta que se quiere procesar para evitar que el sistema cognitivo se sature o desborde su capacidad limitada, dejando fuera la información irrelevante (Dosil, 2004); por lo que supone una variable decisiva para detectar e interpretar las demandas de cada situación deportiva, y posibilita la toma de decisiones (Poveda y Benítez, 2010; Ruiz y Arruza, 2005).

Existe una relación entre activación y grado de atención, pues a medida que el centro de atención se incrementa hacia lo que sucede en el entorno, la atención respecto al sí mismo disminuye. Pero, si el centro de la atención se dirige cada vez más hacia el sí mismo, lo que ocurre en el entorno disminuye e incluso llega a eliminarse. El aumento de la activación por estrés, preocupación por los fallos cometidos o cansancio, hace que la atención se desplace desde el juego hacia la persona, con una influencia negativa en el rendimiento deportivo (González, Garcés de los Fayos y Ortega, 2014; Kent, 2003). Esta relación entre el nivel de activación y el rendimiento deportivo se puede interpretar mediante la clásica teoría de la hipótesis de la U invertida de Yerkes y Dodson (1908), donde el máximo rendimiento tendría lugar en presencia de un nivel de activación óptimo que se encontraría por encima de un nivel de activación más bajo y por debajo de un nivel más elevado que, en ambos casos, por defecto y por exceso, respectivamente, perjudicarían el rendimiento.

El término fatiga se utiliza para referirse a la sensación de cansancio tras un esfuerzo, que puede ser de diversa naturaleza, física o mental, y genera desmotivación para la continuación de ese esfuerzo deportivo (Cárdenas, Conde-González y Perales, 2017). Por tanto, los factores que contribuyen a la fatiga resultante de la actividad física se derivan tanto del esfuerzo físico, como también de la carga mental concomitante y de los resultados de la tarea que se está realizando (Abbiss y Laursen, 2005; Cárdenas, Conde-González y Perales, 2015; St Clair Gibson et al., 2003). No obstante, la fatiga también se deriva de la actividad de naturaleza táctica propia de los deportes de interacción motriz, en los que el deportista realiza un esfuerzo, por un lado, cognitivo, para la toma de decisiones y, por otro, conducente a la autorregulación emocional. En tal caso, después de un esfuerzo físico intenso no existe la incapacidad para continuar la actividad deportiva, sino para hacerlo manteniendo un nivel de rendimiento óptimo (Cárdenas et al., 2015). Existe una sensación de fatiga que se deriva de la capacidad del ser humano para elaborar un constructo mental que resulta de la combinación de múltiples factores psicológicos: la expectativa del rendimiento (predicción basada en la memoria acerca de la fuerza o potencia muscular propia), el grado de activación, la motivación y el estado anímico (López-Chicharro y Fernández-Vaquero, 2006). Esto lleva a la necesidad de asumir que un mismo nivel de esfuerzo físico puede originar sensaciones de fatiga diferentes (Cárdenas et al., 2017).

Los estudios sobre la relación de los procesos cognitivos y los esfuerzos físicos se han basado en el modelo del efecto de la depleción del ego (Baumeister, Vohs y Tice, 2007; Hagger, Wood, Stiff y Chatzisarantis, 2010), entendido como la disminución o el agotamiento de los recursos de un individuo para hacer frente a tareas que impliquen un esfuerzo de autocontrol, ya sea de la atención, cognitivo o emocional. Bajo este efecto un individuo que ha sido sometido a esfuerzos de autocontrol en una primera tarea vería deteriorado su rendimiento en una segunda que implicara este tipo de esfuerzo, aunque la naturaleza de la tarea fuera diferente. Por tanto, existe un reservorio global común de energía y recursos que es independiente de la dimensión específica que sea agotada, lo que deja menos recursos disponibles para la realización de cualquier actividad posterior que requiera autocontrol. Los resultados encontrados muestran claras evidencias de que, el deterioro del rendimiento en tareas propias de los experimentos sobre el efecto de la depleción del ego coincide con percepciones elevadas de fatiga, dificultad y esfuerzo (Kurzban, Duckworth, Kable y Myers, 2013). El efecto de la depleción de recursos ha sido comprobado sobre el rendimiento en tareas con requerimientos físicos (Marcora, Staiano y Manning, 2009; Stults-Kolehmainen 
y Bartholomew, 2012; Bray, Graham, Martin Ginis y Hicks, 2012; Smith, Marcora y Coutts, 2015) y en aquellas en las que de forma paralela se demandaban esfuerzos físicos y mentales (Cárdenas, Perales, Chirosa, Conde, Aguilar y Araya, 2013), donde se señala la contribución de los esfuerzos mentales a la aparición de la fatiga. No obstante, Xu et al. (2014) cuestionan el efecto de la depleción del ego al no ser capaces de replicarlo en sus investigaciones.

Por tanto, el objetivo de la presente investigación fue comprobar si un esfuerzo físico de alta intensidad tiene influencia sobre el rendimiento cognitivo en deportistas entrenados; planteando la hipótesis inicial de que el deportista sometido a una prueba de esfuerzo físico de máxima intensidad disminuye su rendimiento cognitivo.

\section{Método}

\section{Participantes}

La muestra estuvo formada por 9 deportistas de élite de disciplinas de combate en categoría absoluta masculina, que residen en el Centro de Alto Rendimiento de Madrid y cuyas edades estaban comprendidas entre 20 y 24 años ( $M=$ 22.22; DT = 1.79). Todos ellos llevan varios años entrenando como deportistas de alto rendimiento, por lo que están familiarizados con ejercicios que requieren un esfuerzo físico de gran intensidad; participan de forma asidua en competiciones de nivel internacional, con sesiones de entrenamientos diarias en horarios de mañana y tarde.

\section{Instrumentos}

Como prueba perceptiva y de atención, se utilizó el test de Toulouse-Pièron (Toulouse y Pièron, 2004), en la $6^{\mathrm{a}}$ Ed. Revisada en español (Cordero, Seisdedos, González, y De la Cruz 2013). Mediante el instrumento se aprecia la aptitud o capacidad para concentrarse en tareas cuya principal característica es la monotonía junto a la rapidez perceptiva y la atención selectiva y continuada, es decir, puede evaluar la resistencia a la fatiga, la rapidez-persistencia perceptiva y la concentración. El ejemplar del test es una página que contiene 400 cuadraditos ( 10 filas de 40 elementos), de los cuales sólo la cuarta parte, diez en cada fila, son iguales a uno de los dos modelos presentados al principio de la página. La tarea consiste en señalar, durante diez minutos, aquellos cuadraditos que tienen el guion en la misma posición que uno de los dos modelos. El instrumento puede apreciar la aptitud o capacidad para concentrarse en tareas cuya principal característica es la monotonía junto a la rapidez perceptiva y la atención selectiva y continuada; en otras palabras, puede evaluar la resistencia a la fatiga, la rapidez-persistencia perceptiva y la concentración.

Para completar el test de Wingate, se utilizó un cicloergómetro Monark (Ergomedic 828E, Vansbro, Sweeden). Consiste en una prueba física de máxima intensidad de 30 segundos de duración en la que cada sujeto tuvo una carga (Kp) correspondiente al 7.5\% de su masa corporal (Bar-Or, 1987), donde los participantes tenían que alcanzar las máximas rpm en el menor tiempo posible e intentar mantener el mayor número de rpm desde el valor máximo hasta su finalización.

\section{Procedimiento}

En una reunión previa se informó a los deportistas sobre las características del estudio, inscribiéndose de forma voluntaria y firmando un consentimiento informado. El procedimiento de la investigación fue aprobado por el Comité de Ética de la Universidad Alfonso X El Sabio.

La primera fase del estudio se llevó a cabo en una sala del Centro de Alto Rendimiento de Madrid, donde los participantes cumplimentaron individualmente el test de Toulouse-Pierón; los deportistas fueron asignados a un horario a lo largo de la mañana para que acudieran a la sala preparada para el desarrollo de la prueba. En la sala le recibía uno de los investigadores del estudio quien le informaba de las características de la prueba que debía cumplimentar.

Un mes después, los deportistas fueron citados para la segunda fase, que consistió en la realización del test físico de Wingate. Esta prueba fue precedida por un calentamiento que consistía en 10 minutos en cicloergómetro de los cuales los 5 primeros minutos son realizados a ritmo ligero, escogiendo los sujetos la carga y cadencia, seguidos de otros 5 minutos en la que los sujetos pedalean a 60 revoluciones por minuto (rpm) con una carga de 2 kilopondios (Kp), si bien, los últimos 5 segundos de cada minuto realizan un sprint de máxima intensidad. Inmediatamente terminada la prueba física, los participantes volvían a realizar el test de ToulousePierón. Para el desarrollo de esta segunda fase, los deportistas fueron acudiendo de manera individual, según listado de citaciones de la primera fase, al laboratorio de fisiología de la Universidad Alfonso X El Sabio, donde estaba preparado el cicloergómetro para el desarrollo del test de Wingate, y en una sala anexa, con las mismas condiciones que en la primera fase, para la prueba cognitiva. En todo momento estuvieron presentes los investigadores del estudio. 


\section{Resultados}

En la Tabla 1, se aprecian los valores medios y desviaciones típicas de las puntuaciones obtenidas en los dos momentos de aplicación del test Toulouse-Pierón.

Tabla 1. Media y desviación típica de las puntuaciones del test Toulouse-Pierón

\begin{tabular}{lccc}
\hline & Medición & M & DT \\
Test Toulouse-Pierón & LB & 139.44 & 55.527 \\
\cline { 2 - 4 } & Pos-Test & 215.11 & 66.086 \\
\hline
\end{tabular}

Para comprobar si las diferencias entre las puntuaciones obtenidas eran significativas se utilizó la prueba t de Student para muestras relacionadas. Los resultados (Tabla 2) señalan diferencias estadísticamente significativas entre los dos momentos de aplicación ( $t=-8.014 ; p<.001$ ), consiguiendo las puntuaciones más altas cuando realizaron el test de Toulouse-Pierón al finalizar la prueba de Wingate.

Tabla 2. Prueba t de Student

\begin{tabular}{cccc} 
& Análisis & $\mathrm{t}$ & Sig (bilateral) \\
Test Toulouse-Pierón & LB x Post-Test & -8.014 & .000 \\
\hline
\end{tabular}

Como se observa en la Figura 1, en la primera fase hay una tendencia general a tener un nivel atencional más bajo que en la medida de la segunda fase, por lo que es posible afirmar que el test de Wingate ha supuesto un efecto diferencial real.

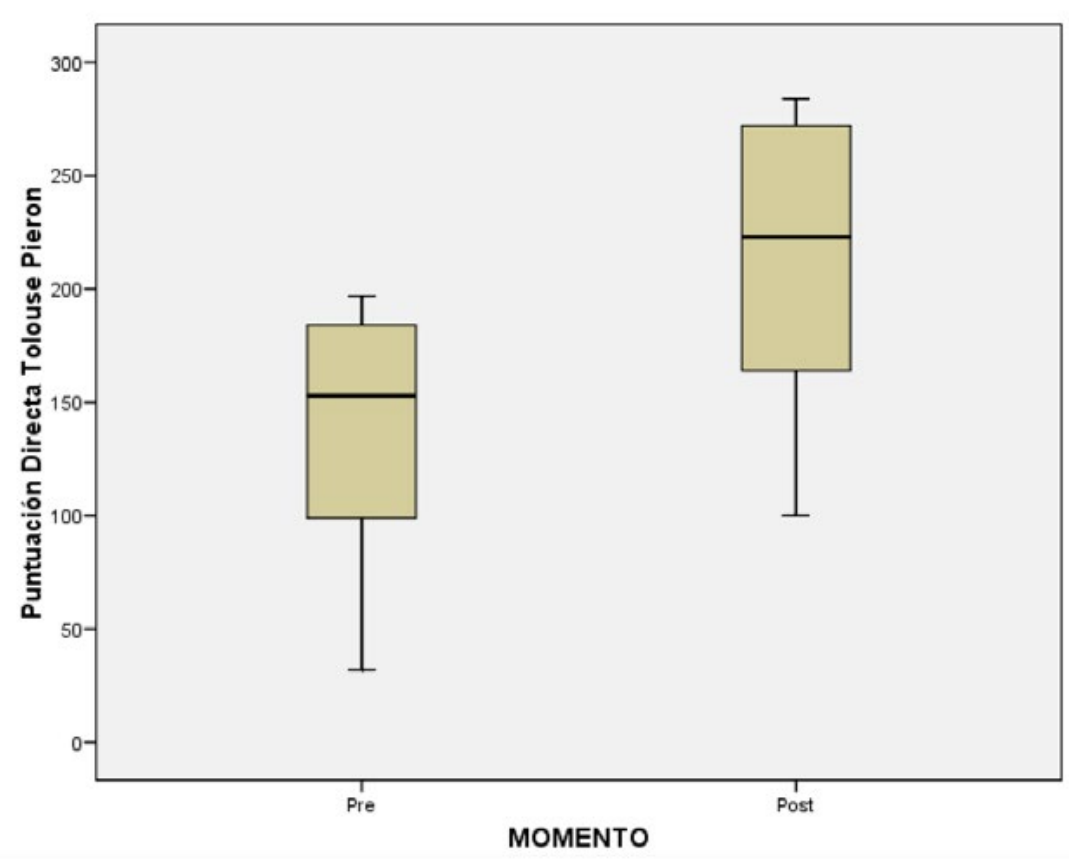

Figura 1. Promedios de los resultados en el Tolouse-Pierón en las dos fases de medición.
El objetivo principal de la presente investigación consistió en comprobar cómo influía la realización de una prueba de máximo esfuerzo físico en el rendimiento cognitivo en una población de deportistas de alto rendimiento. La hipótesis con la que se partía en el trabajo, donde se planteaba que una prueba de esfuerzo máximo disminuía el rendimiento cognitivo, es rechazada, aceptándose que el deportista entrenado aumenta su rendimiento cognitivo cuando su organismo está sometido a una prueba de esfuerzo físico de máxima intensidad.

A partir de los análisis realizados con los resultados del test Tolouse-Pierón se puede observar como la media del grupo incrementó su puntuación en la segunda fase respecto a la primera, diferencias que se corresponden con la línea de investigación de los autores Yerkes y Dodson (1908), explicado a partir de la teoría de la hipótesis de la U invertida, en la cual se plantea que un aumento de la activación produce un mayor rendimiento; este aumento de la activación en deportistas profesionales habituados a realizar actividades que requieren esfuerzo físico intenso, predispone al organismo a situarse en un nivel óptimo de activación ante una exigencia física y mental. Según estos resultados, la relación entre el nivel de activación y los procesos atencionales establece que un deportista que no se encuentra en fatiga tras una prueba de alta intensidad física, es capaz de centrar su atención en lo que sucede en el entorno para tomar decisiones y afrontar las tareas al máximo esfuerzo, mejorando con ello su rendimiento (Rodríguez-Salazar y Montoya, 2006).

Los resultados de nuestro estudio están en la misma línea de conclusiones de otros estudios como los de Xu et al. (2014), que difieren de las conclusiones derivadas de la teoría del efecto de la depleción del ego (Baumeister et al., 2007; Hagger et al., 2010), donde se afirma que se produce un deterioro del rendimiento del individuo que ha sido sometido a una primera tarea con un tipo de esfuerzo físico. Así, los deportistas de élite están preparados para afrontar situaciones que requieren un alto nivel de atención para la toma de decisiones y las respuestas adecuadas, manteniendo un estado de activación elevado.

La modalidad de deporte y nivel de rendimiento influyen en el tiempo de reacción electiva (Martínez, 2003); así, en deportes de combate, los entrenamientos y las competiciones exigen la realización de ejercicios donde se requiere un proceso máximo de atención. La importancia del tiempo de reacción en una actividad deportiva donde un tiempo corto de respuesta ante un estímulo puede establecer una ventaja difícil de recuperar es im- 
prescindible en deportistas con tiempos de reacciones cortos y de mayor duración (Pérez-Tejero, Soto-Rey y Rojo-González 2011).

Como conclusión, en este estudio pudimos comprobar cómo los deportistas entrenados aumentan el rendimiento cognitivo en tareas que requieren atención y toma de decisiones cuando se encuentran en un nivel óptimo de activación. La capacidad de recuperación física de los deportistas de alto rendimiento les posibilita mantener un nivel de activación óptimo, y ejecutar siguientes tareas cognitivas con un rendimiento alto.

Las limitaciones de este estudio se basan principalmente en el escaso número de participantes que constituyen la muestra, que no permiten obtener resultados concluyentes. Además, sería conveniente contar con un grupo de control de participantes que no mantengan un entrenamiento deportivo de alto rendimiento, donde analizar el efecto del esfuerzo físico en los procesos atencionales. Además, habría que establecer un tiempo menor entre la primera fase (evaluación cognitiva) y la segunda (prueba física y evaluación cognitiva) para descartar posibles variables contaminadoras (situaciones vitales del participante, fatiga, circunstancias deportivas, etc.).

A partir de los resultados obtenidos en el presente estudio, se determina que, el rendimiento cognitivo en los procesos atencionales en deportistas de alto rendimiento, experimenta un incremento cuando el deportista está sometido a un nivel de esfuerzo físico intenso, lo que predispone a su organismo a un mejor estado de concentración para el desarrollo de funciones cognitivas como son la toma de decisiones y la rapidez mental.

\section{Referencias}

Abbiss, C. R. y Laursen, P. B. (2005). Models to explain fatigue during prolonged endurance cycling. Sports Medicine, 35, 865898. https://doi.org/10.2165/00007256-200535100-00004

Bar-Or, O. (1987). The Wingate anaerobic test: An update on methodology, reliability and validity. Sports Medicine, 4, 381-394. https://doi.org/10.2165/00007256-198704060-00001

Baumeister, R. F., Vohs, K. D. y Tice, D. M. (2007). The strength model of self-control. Current Directions in Psychological Science, 16, 351-355. https://doi.org/10.1711/j.7467-8721.2007.00534

Bray, S. R., Graham, J. D., Martin Ginis, K. A. y Hicks, A. L. (2012). Cognitive task performance causes impaired maximum force production in human hand flexor muscles. Biological Psychology, 89(1), 195-200. https://doi.org/10.1016/j. biopsycho.2011.10.008

Cárdenas, D., Conde-González, J. y Perales, J.C. (2015). El papel de la carga mental en la planificación del entrenamiento deportivo. Revista de Psicología del Deporte, 24(1), 91-100.
Cárdenas, D., Conde-González, J. y Perales, J.C. (2017). La fatiga como estado motivacional subjetivo. Revista Andaluza de Medicina del Deporte, 10(1), 31-41.

Cárdenas, D., Perales, J. C., Chirosa, L. J., Conde, J., Aguilar, D. y Araya, S. (2013). The effect of mental workload on the intensity and emotional dynamics of perceived exertion. Anales de Psicología, 29, 662-673. https://doi.org/10.6018/analesps.29.3.175801

Cordero, A., Seisdedos, N., González, M. y De la Cruz, V. (2013). Touluse-Piéron revisado- Prueba perceptiva y de atención. Madrid, España: TEA Ediciones.

Dosil, J. (2004). Psicología de la actividad física y del deporte. Madrid, España: McGrawHill.

González, J., Garcés de los Fayos, E. y Ortega, E. (2014). Avanzando en el camino de diferenciación psicológica del deportista. Ejemplos de diferencias en sexo y modalidad deportiva. Anuario de Psicología, 44(1), 31-44.

Hagger, M. S., Wood, C., Stiff, S. y Chatzisarantis, N. L. (2010). Ego depletion and the strength model of self-control: A meta-analysis. Psychological Bulletin, 136, 495-525. https://doi. org/10.1037/a0019486

Kent, M. (2003). Diccionario Oxford de medicina y ciencias del deporte. Barcelona, España: Paidotribo.

Kurzban, R., Duckworth, A., Kable, J. W. y Myers, J. (2013). An opportunity cost model of subjective effort and task performance. Behavioral and Brain Sciences, 36, 661-679. https://doi. org/10.1017/S0140525X12003196

López-Chicharro, J. y Fernández-Vaquero, A. (2006). Fisiología del ejercicio ( $3^{a}$ ed). Madrid, España: Médica Panamericana.

Marcora, S. M., Staiano, W. y Manning, V. (2009). Mental fatigue impairs physical performance in humans. Journal of Applied Physiology, 106, 857-864. https://doi.org/10.1152/japplphysiol.91324.2008

Martínez, O. (2003). El tiempo de reacción visual en el kárate. (Tesis no publicada). Universidad Politécnica de Madrid. Escuela Técnica Superior Arquitectura, Madrid.

Pérez-Tejero, J., Soto-Rey, J. y Rojo-González, J. J. (2011). Estudio del tiempo de reacción ante estímulos sonoros y visuales. Motricidad: European Journal of Human Movement, 27, 149-162.

Poveda, J. y Benítez, J. D. (2010). Fundamentos teóricos y aplicación práctica de la toma de decisiones en el deporte. Revista de Ciencias del Deporte, 6(2), 101-110.

Rodríguez-Salazar, M. C. y Montoya, J. C. (2006). Entrenamiento en el mantenimiento de la atención en deportistas y su efectividad en el rendimiento. Acta Colombiana de Psicología, 9(1), 99-112.

Ruiz, L. M. y Arruza, J. (2005). El proceso de toma de decisiones en el deporte. Clave de la eficiencia y el rendimiento óptimo. Barcelona, España: Paidós.

Santiago, J., Tornay, F., Gómez, E. y Elosúa, M. R (2006). Procesos psicológicos básicos. Madrid, España: Mc.GrawHill.

Smith, M. R., Marcora, S. M. y Coutts, A. J. (2015). Mental fatigue impairs intermittent running performance. Medicine and Science in Sports and Exercise, 47, 1682-1690. https://doi.org/10.1249/ MSS.0000000000000592

St Clair-Gibson, A., Baden, D. A., Lambert, M. I., Lambert, E. V., Harley, Y. X., Hampson, D., ... Noakes, T. D. (2003). The conscious 
perception of the sensation of fatigue. Sports Medicine, 33(3), 167-176. https://doi.org/10.2165/00007256-200333030-00001

Stults-Kolehmainen, M. A. y Bartholomew, J. B. (2012). Psychological stress impairs short-term muscular recovery from resistance exercise. Medicine and Science in Sports and Exercise, 44, 2220-2227. https://doi.org/10.1249/MSS.0b013e31825f67a0

Toulouse, E. y Piéron, H. (2004). Toulouse-Piéron, prueba perceptiva y de atención. Madrid, España: TEA Ediciones.
Xu, X., Demos, K. E., Leahey, T. M., Hart, C. N., Trautvetter J., Coward P., ... Wing, R. R. (2014). Failure to replicate depletion of self-control. PLoS One, 9(10), e109950. https://doi.org/10.1371/journal. pone.0109950

Yerkes, R. M. y Dodson, J. D. (1908). The relation of strength of stimulus to rapidity of habit formulation. Journal of Comparative and Neurological Psychology, 13, 459-482. 